\title{
Applications of Kite Flap in Reconstruction of Cheek Defects after Tumor Excision
}

\author{
Ali Ebrahimi ${ }^{1}$, Mohammad Hosein Kalantar Motamedi ${ }^{1}$, Ehsan Shams Koushki ${ }^{1}$, Nasrin Nejadsarvari ${ }^{2}$ \\ ${ }^{1}$ Trauma Research Center, Baqiyatallah University of Medical Sciences, Tehran, Iran; ${ }^{2}$ Imam Khomeini Hospital, Tehran University of \\ Medical Sciences, Tehran, Iran
}

\begin{abstract}
Citation: Ebrahimi A, Motamedi MHK, Koushki ES, Nejadsarvari N. Applications of Kite Flap in Reconstruction of Cheek Defects after Tumor Excision. Maced J Med Sci. 2012Oct 15; 5(3):313316. http://dx.doi.org/10.3889/MJMS.19575773.2012.0233.

Key words: kite flap; island flap; cheek reconstruction; neoplasm; Basal cell carcinoma.

Correspondence: Mohammad Hosein Kalantar Motamedi. P.O.Box: 1436614313 Tehran. Telephone Iran: +9821-88053766; Fax: $+9821-88053766$.

traumabmsu@ymail.com

Received: 31-Oct-2011; Revised: 05-Mar-2012 Accepted: 08-Mar-2012; Online first: 03-Sep-2012

Copyright: (c) 2012 Ebrahimi A. This is an openaccess article distributed under the terms of the Creative Commons Attribution License, which permits unrestricted use, distribution, and reproduction in any medium, provided the original

Competing Interests: The author have declared that no competing interests exist.
\end{abstract}

\section{Abstract}

Objectives: The main aim of this study was to present our experience with $\mathrm{V}-\mathrm{Y}$ island flaps in reconstruction of the cheek defects after tumor excision in 3 facial zones.

Patients and methods: 40 patients with cutaneous carcinomas of the cheek in 3 facial zones (17 SubOrbital, 19 Bucco-Mandibular and 4 Pre-Auricular) resulting from skin tumor excision were reconstructed using V-Y flaps from July 2009- June 2011.

Results: 40 patients with small to medium-sized defects $(3-6 \mathrm{~cm})$ ranging from 25 to 70 years were treated. Of these 40 patients, 17 were men $(42.5 \%)$ and 23 were women (57.5\%). We had no complications and the functional and aesthetic outcomes were quite acceptable. No recurrence of malignant skin tumor was observed to date (mean $1.5 \mathrm{yrs}$.).

Conclusions: The kite flap is one of the methods for reconstruction of small to medium-size defects in different zones of the cheek. This method has aesthetic and functional superiority in comparison with skin grafts or other local flaps in the cheek.

\section{Introduction}

Cutaneous carcinomas of the cheek may involve the epidermis, dermis, and subcutaneous tissues that overlie the superficial musculo-aponeurotic system (SMAS) (in fact skin cancer can involve even deeper structures of the face) [1, 2]. The cheek has bordering subunits that help dictate the primary goal in cheek reconstruction: avoidance of deformation of the surrounding structures. Surgeons must recognize the safety margins of the adjacent structures, including the lower eyelid, nasal ala, and lip, to adhere to the priority goals of first preserving function and second achieving cosmesis [3]. Surgical reconstruction of the cheek remains a challenging aspect of facial plastic and reconstructive surgery.

There are several reconstructive options after excision of skin tumors in this region of face, including primary repair, skin grafts, local flaps, regional flaps, distant and free flaps. Local flaps including $V-Y$ 
advancement flaps are standard methods for cheek defects.

In this article, we explain our experiences using $\mathrm{V}-\mathrm{Y}$ flaps in the reconstruction of small to moderate sized cheek defects after tumor excision.

\section{Patients and Methods}

Forty patients with skin defects (17 sub-Orbital, 19 Bucco-Mandibular and 4 Pre-Auricular) resulting from skin tumor excision were reconstructed using V-Y flaps from July 2009 - June 2011. In these patients, cheek tumors (Basal Cell Carcinoma or Squamous Cell Carcinoma) were excised and safe margin confirmed by frozen section; reconstruction of the small to moderate sized $(3-6 \mathrm{~cm})$ cheek defects in different zones were done by v-y plasty. All operations were performed under sedation and local anesthesia.

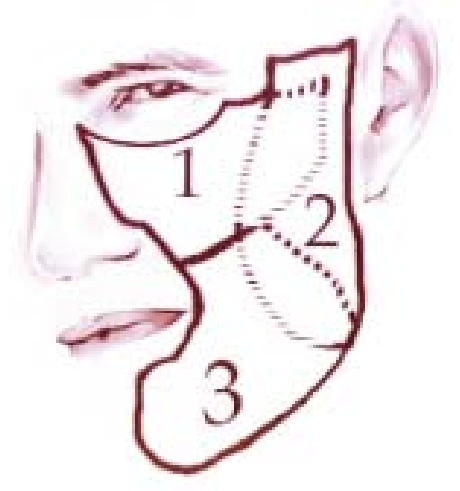

Figure 1: 1) Sub-Orbital zone 2) Pre-Auricular zone 3) Bucco-Mandibular zone Areas that marked with dotted lines are overlapped zones that are common between two zones.

Safe margins were $4-5 \mathrm{~mm}$ for BCC and 6$10 \mathrm{~mm}$ for SCC before the frozen section report. The length of flap was twice the diameter of the base. To reduce tension of the skin, we repaired subcutaneous tissues with absorbable suture. Blood supplies of these flaps were preserved by identifying perforating arteries in the central part of flap from subcutaneous arterial networks In most cases flap design was parallel to resting skin tension lines.

Permanent sutures were removed at 5 and 7 days postoperatively. Patients were followed monthly thereafter.

\section{Results}

Fourty (40) patients ranging from 25 to 70 years (mean: 52.9) 17 were men (42.5\%) and 23 were women (57.5\%). The V-Y flap in different zones of cheek is an aesthetic method to reconstruct small to medium-size defects $(3-6 \mathrm{~cm})$. Pathological diagnosis of lesions
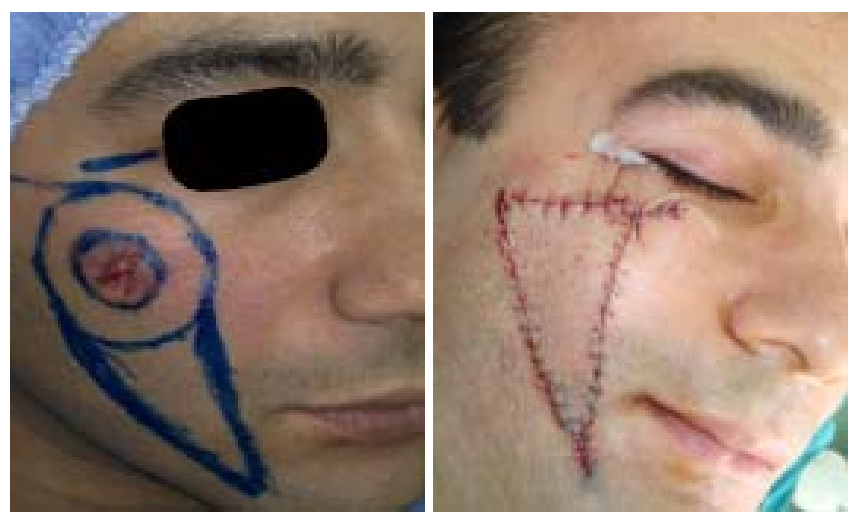

Figure 2: Basal Cell Carcinoma lesion in Sub-Orbital zone of the cheek (Left) Design of the flap. (Right) Closure of the wound with no distortion of the surrounding structures.

included Basal Cell Carcinoma (n: 32), Squamous Cell Carcinoma (n: 8). Defect sizes after the excision of tumors ranged from $2 \times 2 \mathrm{~cm}$ to $6 \times 6 \mathrm{~cm}$ (mean: $3.72 \times 4.35 \mathrm{~cm}$ ) including skin and subcutaneous tissue and in some cases partial mimetic muscles. In all cases and in the three zones of the cheek, favorable cosmetic and functional results were obtained with a single-stage procedure that did not require secondary revisions.

In all patients examined scars were minimal after operation. We had no complications such as
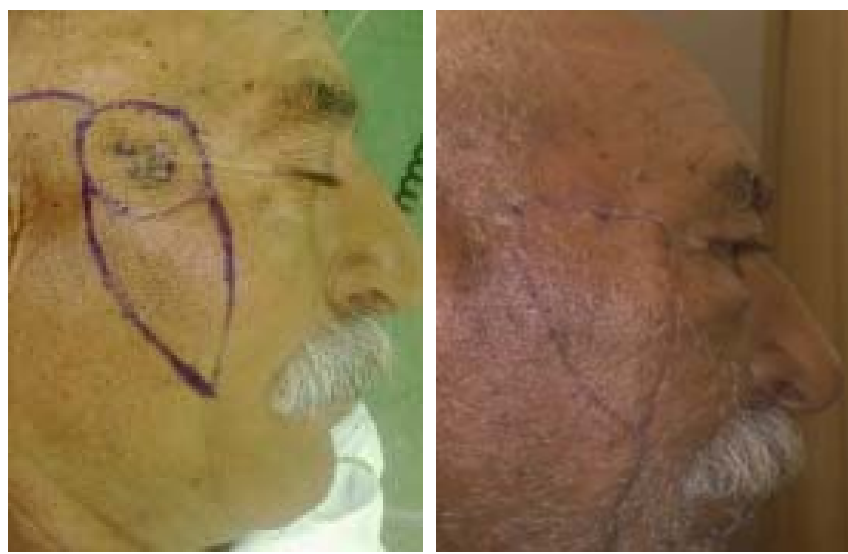

Figure 3: Basal Cell Carcinoma lesion in Pre-Auricular zone of the cheek. (Left) Design of the flap. (Right) Three months post-operatively. 
hematoma, infection, visible dog-ear, pin-cushioning, ischemia or necrosis of flap. Neither deformities of the flap nor abnormal scar were seen. Scars were well concealed. These flaps had similar thickness and texture to adjacent skin after several months (Figures 2, 3). No recurrence of malignant skin tumor was observed during the $1.5 \mathrm{yr}$. period of follow-up (Table 1).

Table1: Summary of collected data in defects reconstructed with V-Y flaps.

\begin{tabular}{lccccc}
\hline Cheek Zones & Number of Patients & Age & Sex(F/M) & Defect Size & Type of Tumour \\
\hline Bucco-Mandibular & 19 & $30-70$ (mean:50.1) & $12 / 7$ & $4.26 \times 4.68 \mathrm{~cm}$ & BCC : $19(100 \%)$ \\
\hline Sub-Orbital (SO) & 17 & $25-70$ (mean:50.1) & $11 / 6$ & $2.76 \times 3.65 \mathrm{~cm}$ & BCC: $13(76.4 \%)$ \\
\hline Pre-Auricular (PA) & 4 & $30-70$ (mean: 58.7$)$ & $0 / 4$ & $5.25 \times 5.75 \mathrm{~cm}$ & SCC: $4(23.6 \%)$ \\
\hline Total & 40 & $25-70$ (mean:52.9) & $23 / 17$ & $3.72 \times 4.35 \mathrm{~cm}$ & BCC $32(80 \%)$ \\
\hline
\end{tabular}

BCC: Basal Cell Carcinoma; SCC: Squamous Cell Carcinoma.

\section{Discussion}

Cheek defects can have a traumatic or congenital origin or occur after ablative surgery for skin cancers. The reconstruction of such defects, being one of the most frequently performed tasks by plastic surgeons, can sometimes be challenging $[4,5]$.

There are several reconstructive options after excision of skin tumors of the cheek, including primary repair, skin grafts, local different flaps, regional flaps, distant and free flaps. The choice of reconstructive procedure is dependent upon several factors, including size, location, involvement of deeper structures, etc.

Primary repair is used in small defects $<6 \mathrm{~cm}$ when there is no tension on the edges of the defect. For larger defects there are several local flaps for cheek reconstruction including Rhomberg transposition flap, advancement flap, rotation-advancement flap (Mustarde flap), v-y advancement (kite flap). Another method in cheek reconstruction is skin grafting; Limitations of grafts include donor site morbidity, patchy appearance and less similarity in contour, texture or color.

For larger cheek defects $(>6 \mathrm{~cm})$, we need some type of regional or distant flap. Free flaps are applicable for complex soft tissue and bone defects or through and through defects. Due to the unique characteristics of the cheek subunit, few complex local flap designs can be used to reconstruct the vast majority of defects. Obviously, no one method can be used for all defects.

However, management is usually straight forward due to availability of excess tissue laterally and inferiorly [4].

Most of the local skin cancers of the head and neck are either squamous or basal cell carcinoma. Large full thickness defects can result from resection of these tumors, especially if the lesions are adherent to bone.

Reconstruction should preferably be with local tissue to achieve the best cosmetic results [6]. Advancement flaps depend on the advancement of the surrounding tissue along a linear axis to close a defect. The advancement of two skin edges from a fusiform skin excision represents the simplest of advancement flap designs. Classically, advancement flaps have a lengthto-width ratio of $1: 1$ or $2: 1$.

Advancement flaps often create standing cutaneous deformities or "dog-ear" deformities, which must also be addressed as part of the reconstruction plan [7]. The V-Y flap is one type of advancement flap. The face is one of the most commonly affected sites by skin tumors. Resultant defects may have various patterns and include one or more soft and/or hard tissues. The ideal facial reconstruction should provide a good color and texture harmony with adjacent tissues. Minimal donor-site morbidity with well-hidden scar is also desirable.

One of the aesthetic local flaps in the cheek reconstruction is the kite flap that should be designed parallel to relaxed skin tension lines for best functional and aesthetic results, designing of the flap not parallel to relaxed tension lines may also have an acceptable result. Although full-thickness skin grafting and numerous free flaps can be used, these alternatives are usually difficult to match the desirable criteria in facial reconstruction [8]. Various surgical options have been described for repair of these conspicuous defects. Individual factors, including patient preference, overall patient health, and characteristics of the defect, will help to define the appropriate surgical approach. Regardless of the method chosen, the optimal result will require correction of both functional and cosmetic abnormalities [9].

A great number of local flaps, pedicled flaps and micro-vascular free flaps have been employed over the years for the reconstruction of mid-facial defects. Nevertheless, reconstruction of the mid-face remains a challenging and still varied problem due to the different defects and the complex three-dimensionality of the region [10]. In our past experience with regional flaps in the treatment of maxillofacial soft-tissue injuries, initial 
healing of the flaps was favourable in $76 \%$ of the patients. However, postoperative discharge from the suture sites was seen in $24 \%$ of the patients [11].

In the present study we did not have any complications as these were clean wounds. The $V-Y$ flap may be an ideal option for facial cutaneous reconstruction [12]. However, the movement of the traditional $V-Y$ flap is sometimes limited because its pedicle only allows for rotation or advancement. Several modifications have been proposed to maximize $\mathrm{V}-\mathrm{Y}$ advancement movement by ameliorating it's undermining, designing an extended limb, or using a bilateral subcutaneous pedicle, a single lateral pedicle or parallel release incisions. Optimal aesthetic reconstruction of facial defects is largely dependent on the availability of regional donor tissues sharing similar color, texture, sebaceous quality, and thickness with the defect tissues.

Conclusion: We found the kite flap to be an effective method for reconstructing small to mediumsized defects of the cheek. This method has aesthetic and functional results. Furthermore, it has no donor site morbidity in contrast to skin grafts.

\section{References}

1. Furr MC, Wang TD. Complex local flap design in cheek reconstruction. Operative Techniques in Otolaryngology-Head and Neck Surgery. 2011;22(1):53-8.

2. Dobratz EJ, Hilger PA. Cheek Defects. Facial Plastic Surgery Clinics of North America. 2009;17(3):455-67.

3. Li JH, Xing X, Liu HY, Li P, Xu J. Subcutaneous island pedicle flap: variations and versatility for facial reconstruction. Ann Plast
Surg. 2006 Sep;57(3):255-9.

4. Summers BK, Siegle RJ. Facial cutaneous reconstructive surgery: Facial flaps. Journal of the American Academy of Dermatology. 1993;29(6):917-41.

5. Demirseren ME, Afandiyev K, Ceran C. Reconstruction of the perioral and perinasal defectswith facial artery perforator flaps. Journal of Plastic, Reconstructive \& Aesthetic Surgery. 2009;62(12):1616-20.

6. Khan HA, Niranjan NS. Four V-Y islanded flap reconstruction of full thickness defect of chin and labial sulcus. British Journal of Plastic Surgery. 2004;57(3):278-81.

7. Patel KG, Sykes JM. Concepts in local flap design and classification. Operative Techniques in Otolaryngology-Head and Neck Surgery. 2011;22(1):13-23.

8. Tan O. An algorythmic approach to restoration of the frontonaso-periorbital skin defects using the forehead flaps. Journal of Cranio-Maxillofacial Surgery. 2010;38(1):11-8.

9. Cook TA, Davis RE. Cheek reconstruction. Operative Techniques in Otolaryngology- Head and Neck Surgery. 1993;4(1):31-6.

10. Dallan I, Lenzi R, Sellari-Franceschini S, Tschabitscher M, Muscatello L. Temporalis myofascial flap in maxillary reconstruction: anatomical study and clinical application. Journal of Cranio-Maxillofacial Surgery. 2009;37(2):96-101.

11. Motamedi MHK, Behnia $\mathrm{H}$. Experience with regional flaps in the comprehensive treatment of maxillofacial soft-tissue injuries in war victims. Journal of Cranio-Maxillofacial Surgery. 1999;27(4):256-65.

12. Li J-h, Xing X, Li P, Xu J. Transposition movement of $\mathrm{V}-\mathrm{Y}$ flaps for facial reconstruction. Journal of Plastic, Reconstructive \& Aesthetic Surgery. 2007;60(11):1244-7. 